International Journal of Embedded Systems and Applications (IJESA) Vol.2, No.3, September 2012

\title{
A NEW REVOLUTIONARY INFRARED LIFE DETECTION SYSTEM USING ATMEgA168
}

\author{
Ashutosh Gupta ${ }^{1}$ and Betsy Thomas ${ }^{2}$ \\ ${ }^{1}$ Assistant Professor, Department of Electronics and Communication Engineering, Amity \\ University, Noida, India \\ ashugupta09@gmail.com \\ ${ }^{2}$ M.Tech Scholar, Department of Electronics and Communication Engineering, Amity \\ University, Noida, India \\ betsy.jasonegmail.com
}

\begin{abstract}
A new sensitive infrared life-detection system which can be used to locate and obtain valuable information regarding human subjects which are buried under earthquake rubble has been developed. This system can detect the distance at which the human subject is detected and the heartbeat signals of the person under the earthquake rubble can be measured. In this system, an infrared sensor comprising of a light emitting diode, adjacent to a phototransistor is being used. When an infrared beam is aimed at a portion of earthquake rubble covering a human being, the beam can penetrate the rubble to reach the human subject. Distance measurement is carried out using an infrared sensor, consisting of one infrared LED and a pair of silicon phototransistors. By using high intensity, long range infrared distance sensors, the distance from a human subject can be precisely and reliably measured and it can be indicated to the rescue operators.
\end{abstract}

Infrared light is absorbed well in blood and weakly absorbed in tissue. The IR light reflected back from the skin of a human subject on account of blood passage is captured by the detector. This reflected light has intensity variations which occur as the blood volume changes in the tissue and it results in voltage variations. This voltage variation determines the heart rate. After measuring the heart rate, it can be indicated on the monitor and immediate action can be taken for rescuing the person. It has been medically proved that a person can be rejenuvated back to life if the heart rate is above $60 \mathrm{bpm}$.

A hardware prototype of the life detection system has been developed and experimental results show that the proposed method is cost effective and efficient method which not only detects life signals but also the identification of people in a given area, to facilitate rescue team operations in case of emergencies. By the advent of this system, the world death rate as a cause of natural disasters like earthquake may decrease to greater extent.

\section{KEYWORDS}

Life Detection, Earthquake rubble, Infrared, distance measurement, Heartbeat detection

\section{INTRODUCTION}

Some existing methods for detecting human victims buried under earthquake rubble is the utilization of dogs or some optical devices. These existing devices are ineffective in recovering human victims lying much below a few feet, especially for the cases where the victims are completely trapped below or very weak to respond to the signal sent by the rescuers. Thus, there is great demand for constructing a new sensitive life-detection system which can be used to locate human victims trapped deep under earthquake rubble. Especially, the system needs to be sensitive enough to detect the heartbeat signals of victims. We have constructed a sensitive life-detection DOI : $10.5121 /$ ijesa.2012.2313 
International Journal of Embedded Systems and Applications (IJESA) Vol.2, No.3, September 2012

system for such purposes using infrared radiation. In this paper we will describe an infrared lifedetection system.

The basic physical principle for the operation of infrared life-detection system is rather simple. When an infrared beam is aimed at a portion of earthquake rubble covering a human being, the beam penetrates the rubble to reach the human subject. By using high precision, long range infrared distance sensors comprising of powerful LED, the distance from a human subject can be precisely and reliably measured and it can be indicated to the rescue operators.

Information about the location of buried person and other details regarding on the condition of the human subject, would be of great value for the rescue personnel. This would help in reducing the time of operation and thereby, help to save more lives. Hence there is a need to construct a cost effective life detection system which can detect buried victims under earthquake most efficiently and as possible in a short time. Infrared (IR) sensors are extensively used for measuring distances. It uses reflected light intensity to estimate the distance from an object [1-3]. Their inherently fast response is attractive for enhancing the real-time response of a mobile robot [4]. Some IR sensors described in the bibliography are based on the measurement of the phase shift, and offer medium resolution from $5 \mathrm{~cm}$ to $10 \mathrm{~m} \mathrm{[5],} \mathrm{but} \mathrm{these} \mathrm{are} \mathrm{very} \mathrm{expensive.}$

The infrared life-detection system we constructed has four major components: 1) a infrared LED source which generates and distributes infrared signals to various infrared components; 2) a photo transistor and a Light Dependent Resistor (LDR) which is used to detect the IR signals; 3 ) a quad Opamp in non inverting mode as amplifier and comparator; and 4) a ATMega168 Microcontroller which controls the system and provides the output on an 16x2 LCD as the output signal. The system is operated by a portable battery unit.

\section{CIRCUIT DESCRIPTION OF THE SYSTEM}

The novel idea of this paper is to develop efficient and cost-effective life detection and distance measurement system for detecting life under debris. The prototype developed consists of three sections:

1) Input section consisting of (i) IR detection for distance measurement (ii) Heart rate detection

2) Processing section using Microcontroller ATMEGA168

3) Output section consisting of $16 \times 2$ LCD display.

The system consists of infrared transmitter and receiver units. The circuit works in two modes that is as IR distance meter and life detection mode. The mode is decided through a micro-switch which is connected to the input pin of microcontroller. When the switch is pressed for odd number of times, the system remains in mode 1 and for when pressed for even number of times the system is in mode 2. According to the mode selected, the mode LED's are on/off that is either green or red.

\subsection{Block Diagram}

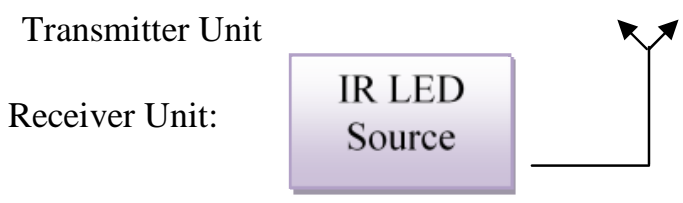




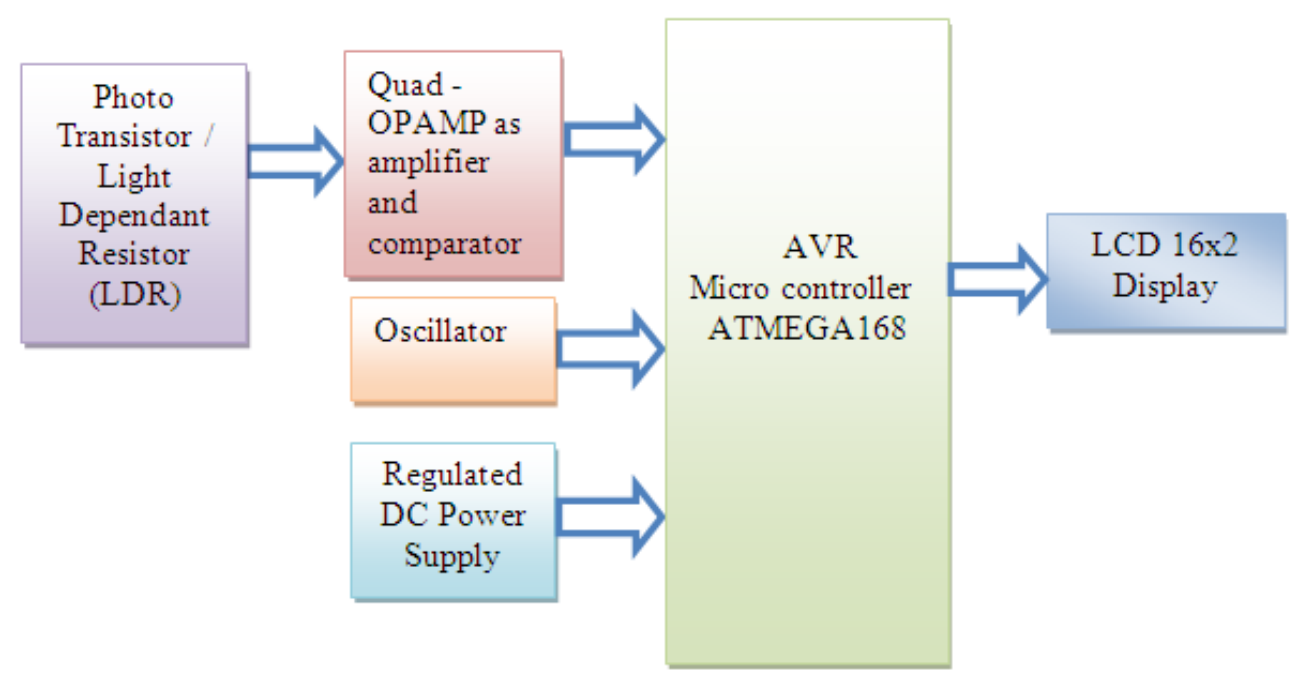

Fig.1 Block Diagram of the system

\subsection{Principle of Working}

There are main sections in the prototype designed:

$>$ IR Based Distance Meter

$>$ Life Detection Circuit

$>$ Processing through ATMEGA168 Microcontroller

\subsection{IR Based Distance Meter}

In this module, an IR source is designed, which represents the human being emitting IR (between 5 to 15 lumens). An IR transmitter is designed through an IR led with a series resistance. 9V battery is connected across the transmitter. The IR receiver section contains a photodiode which receives the IR rays and on the basis of IR intensity provides variation in voltage. The analog voltage obtained is in between its maximum and minimum operating range. This analog variation of voltage is fed to the microcontroller.

\subsection{Life Detection Circuit}

In this section, the basic principle used is on the change in blood flow when the heart functions. A laser source and a LDR (Light Dependant Resistor) are used in this block. In the prototype model developed, a finger is placed in between the laser source and the LDR. If there is change in intensity of light, due to the change in concentration of blood across the finger when the heart pumps blood, the light intensity received also changes. This indicates that the human is alive else when the concentration of blood remains constant, it indicates that the human is dead.

The variation is in some milli-volts which is minimal; hence a non inverting amplifier has been designed using op-amp to amplify the signal. IC LM324-Quad Op-Amp IC is used here. This amplified signal is fed to another op-amp used in comparator mode to change the output into digital format depending upon the threshold set through the preset. The digital output is then fed to the digital pin of the microcontroller. 
International Journal of Embedded Systems and Applications (IJESA) Vol.2, No.3, September 2012

\subsection{Processing through ATMega168 Microcontroller}

The processing is done through ATMEGA168 microcontroller. The input from the IR receiver is fed to the analog input pin of the controller which is converted into discrete levels through 10-bit inbuilt ADC. On the basis of these discrete levels, the system is calibrated for the distance. The maximum discrete level is 1024 and minimum is 0 . Similarly, there will be a maximum and minimum distance also. Likewise in the life detection circuit, the digital input is fed to digital pin of the controller on the basis of which, the detection of whether the human is alive or dead is detected. All the system outputs are managed on a 16X2 LCD through its interface with the microcontroller.

\subsection{Software used}

The processing of inputs and output generation is in accordance with the programming of the microcontroller to take input and generate outputs. The microcontroller is programmed in arduino compiler using $\mathrm{C}++$ logic.

\section{HARdWARe Prototype}

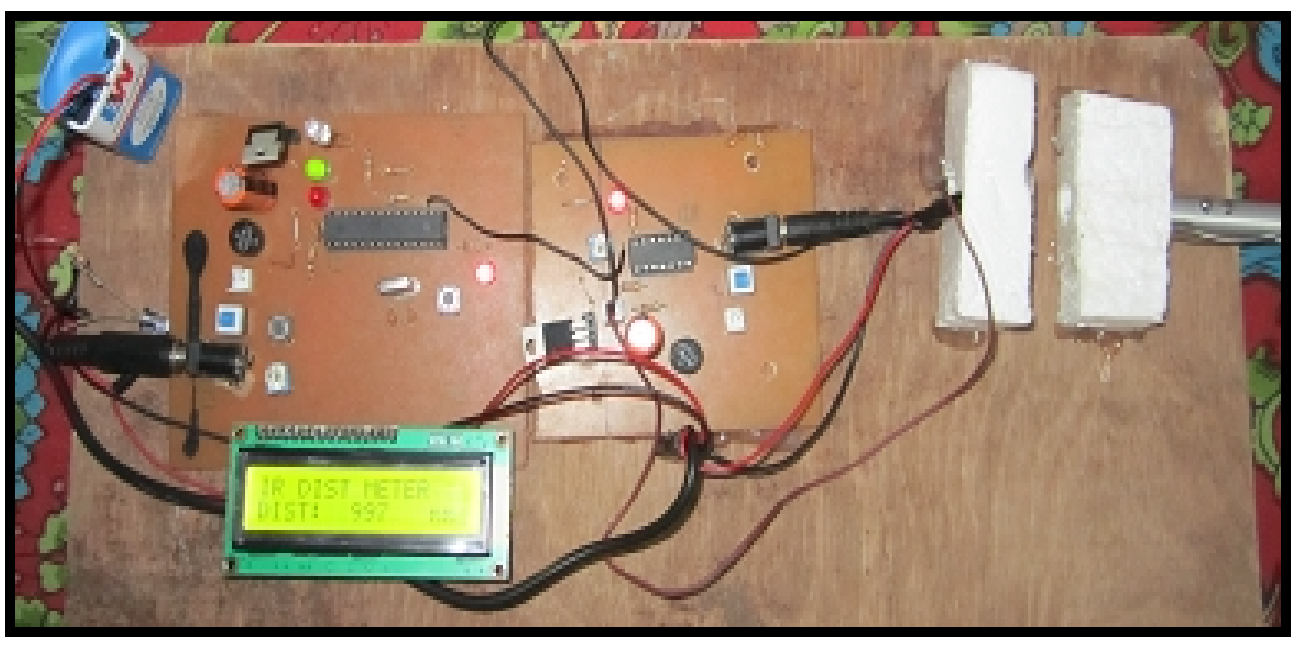

Fig 2. Infrared Life Detection using ATMega168

\section{RESUlts ON LCD}

For Distance measurement:

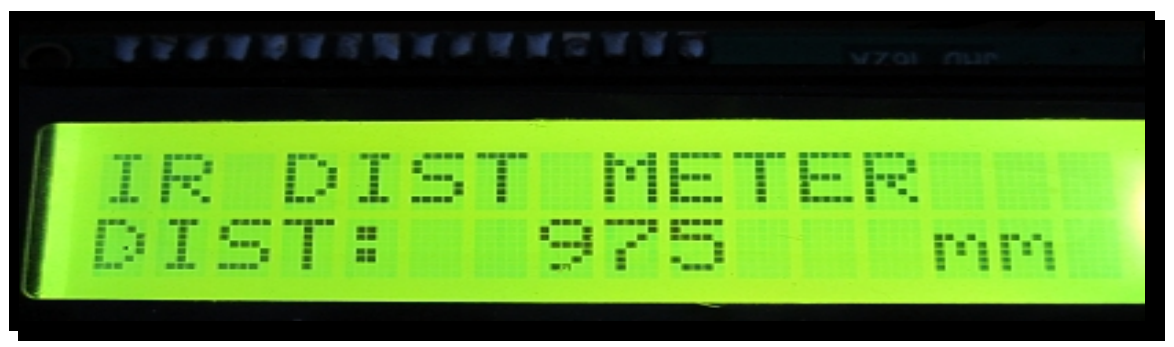


For Heartbeat detection:

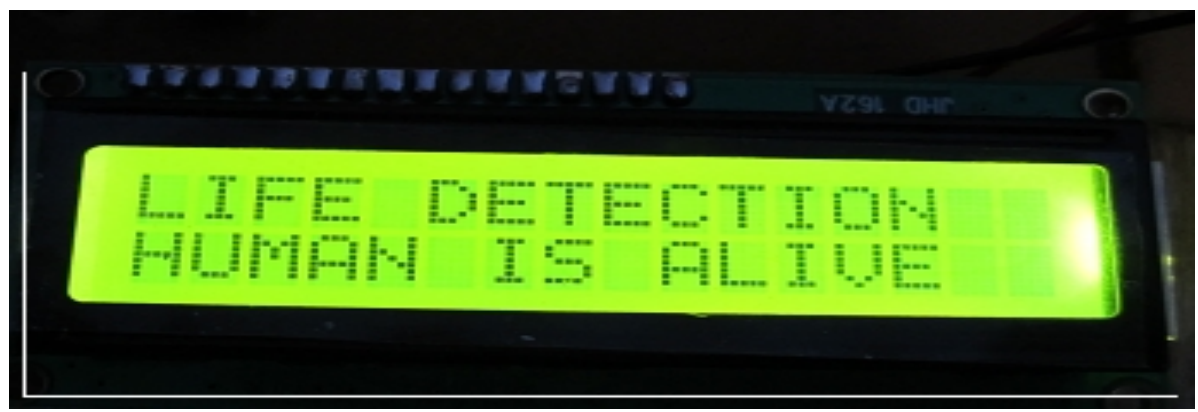

\section{Conclusions}

A new revolutionary sensitive life-detection system using infrared radiation for locating human subjects under earthquake rubble or hidden behind various barriers has been constructed. This system works in two modes that is as IR distance meter and life detection mode. The mode is selected through a micro-switch which is connected to the input pin of the microcontroller.

A hardware prototype of the infrared life detection system has been developed and experimental results show that the proposed method is an efficient method which not only detects life signals but also the identification of people in a given area, to facilitate rescue team operations in case of any emergencies.

\section{ACKNOWLEDGEMENTS}

The authors would like to thank Amity University for providing us with the opportunity to unleash our ideas and turn it into fine work. They would also like to thank Dr. M.K. Dutta (Head Of Department-ECE) under whose guidelines we could exhibit a spectral view on 'A new revolutionary infrared life detection system using ATMEGA168'.

\section{REFERENCES}

[1] V. Colla, A.M. Sabatini, "A composite proximity sensor for target location and color estimation," IMEKO Sixth International Symposium on Measurement and Control in Robotics, Brussels, 1996, pp. $134-139$.

[2] L. Korba, S. Elgazzar, T. Welch, “Active infrared sensors for mobile robots,” IEEE Transactions on Instrumentation and Measuremen, vol. 2(43), 1994, pp. 283-287.

[3] A.M. Sabatini, V. Genovese, E. Guglielmelli, “A low-cost, composite sensor array combining ultrasonic and infrared proximity sensors, IEEE/RSJ International Conference on Intelligent Robots and Systems (IROS), Pittsburgh, PA, vol. 3, 1995, pp. 120-126..

[4] H.R. Everett, Sensors for Mobile Robots, AK Peters, Ltd., Wellesley,MA, 1995.

[5] G. Benet, F. Blanes, J.E. Simo, P. Perez, "Using infrared sensors for distance measurement in mobile robots,” Journal of Robotics and Autonomous Systems, vol. 10, 2002, pp. 255-266. 
International Journal of Embedded Systems and Applications (IJESA) Vol.2, No.3, September 2012

[6] Chen, K. M., Y. Huang, J. Zhang, and A. Norman, Microwave life-detection systems for searching human subjects under earthquake rubble and behind barrier," IEEE Trans. Biomed. Eng., Vol. 27, $105\{114,2000$.

[7] Akihiro Watanabe, et al.: "Lane detection for a Steering Assistance System," Proceedings of the IEEE Intelligent Vehicles Symposium, pp. 159-164, 2005. [3] Yoshikatsu Kimura, et al.: "Stereo vision for obstacle detection," Proceedings of 13th World Congress on Intelligent Transport Systems, no. 1082, 2006.

\section{Authors}

\section{Ashutosh Gupta}

Received B.E. (ECE) and M.Tech. in Vlsi Design \& Embedded systems from Guru Jambheshwar University of Science \& Technology, Hisar in 2006 and 2008, respectively. Having experience from Digital Systems Group, Central Electronic Engineering Research Institute, Pilani, India. Presently working in Amity University Noida. His field of interest are Embedded Systems, FPGA based Reconfigurable computing systems.

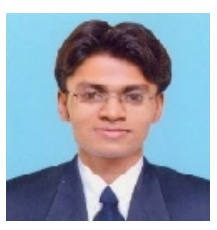

\section{Betsy Thomas}

Received B.E. (EEE) from Bharathiyar University, Coimbatore in 2004 and currently pursuing M.Tech in Embedded Systems from Amity University, Noida. Having 4.8 years of work experience in the field of VLSI board design, development and testing from Wipro Technologies, Bangalore, India. Her field of interest are Embedded systems design and Real time operating system.

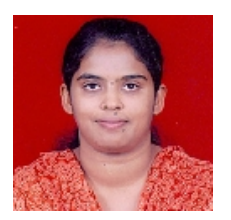

